\title{
A Characterization of Boundedness of Fractional Maximal Operator with Variable Kernel on Herz-Morrey Spaces
}

\author{
Lutfi Akin* \\ Department of Business Administration, Mardin Artuklu University, Mardin, Turkey
}

Received 21 November 2018; Accepted (in revised version) 21 February 2019

\begin{abstract}
A significant number of studies have been carried out on the generalized Lebesgue spaces $L^{p(x)}$, Sobolev spaces $W^{1, p(x)}$ and Herz spaces. In this paper, we demonstrated a characterization of boundedness of the fractional maximal operator with variable kernel on Herz-Morrey spaces.
\end{abstract}

Key Words: Variable exponent, herz space, operator theory.

AMS Subject Classifications: 42B25, 42B35, 47B38

\section{Introduction}

In 1991, Kovacik and Rakosnik introduced variable exponent Lebesgue spaces and Sobolev spaces as a new method for dealing with nonlinear Dirichet boundary value problem [9]. Then, variable problem and differential equation with variable exponent are intensively developed. In recent years, many researchers have been interested by the theory of the variable exponent function space and its applications $[16,17]$. For example, the compactness of Hardy spaces with weighted and variable exponent Lebesgue spaces was introduced by author [11]. Fractional integral on Herz-Morrey spaces with variable exponent were introduced by authors [7] and [10]. The boundedness of fractional integral with variable kernel on variable exponent Herz-Morrey spaces was given by [1]. Boundedness of fractional integral with variable kernel and their commutators on variable exponent Herz spaces was given by [2]. Boundedness of fractional Marcinkiewicz integral with variable kernel on variable exponent Morrey-Herz spaces was given by [3]. We also note that Herz-Morrey spaces with variable exponent are generalization of Morrey-Herz spaces [8] and Herz spaces with variable exponent $[8,13]$. Our main goal is to give a characterization on boundedness of the fractional maximal operator with variable kernel from $M H_{q_{1}, p_{1}(\cdot)}^{\beta, \alpha}\left(R^{n}\right)$ to $M H_{q_{2}, p_{2}(\cdot)}^{\beta, \alpha}\left(R^{n}\right)$.

${ }^{*}$ Corresponding author. Email address: lutfiakin@artuklu.edu.tr (L. Akin) 
Let $0<\rho<m, \Theta \in L^{\infty}\left(R^{m}\right) \times L^{n}\left(S^{m-1}\right)$ and for $x \in R^{m}, \Theta(X, \cdot) \in L^{n}\left(S^{m-1}\right),(n \geq 1)$ is homogenous on $R^{m}, S^{m-1}$ denote the unit sphere in $R^{m}$. If

a) For $x, t \in R^{m}, \Theta(x, \alpha t)=\Theta(x, t)$,

b) $d v\left(t^{\prime}\right)$ is an element of area in the $S^{m-1}$ and

$$
\|\Theta\|_{L^{\infty}\left(R^{m} x L^{n}\left(S^{m-1}\right)\right)}=\sup _{x \in R^{m}}\left(\int_{S^{m-1}}\left|\Theta\left(x, t^{\prime}\right)\right|^{n} d v\left(t^{\prime}\right)\right)^{\frac{1}{n}}<\infty .
$$

We need the further assumption for $\Theta(x, t)$. For $t \in R, \Theta(\cdot, t) \in L^{\infty}\left(R^{m}\right)$. It satisfies

$$
\int_{S^{m-1}}\left|\Theta\left(x, t^{\prime}\right)\right|^{n} d v\left(t^{\prime}\right)=0, \quad \forall x \in R^{m} .
$$

For $n \geq 1$, we say $\Theta(x, t)$ satisfies

$$
\int_{0}^{1} \frac{w_{n}(\lambda)}{\lambda} d \lambda<\infty
$$

where $w_{r}(\lambda)$ denote the integral modulus of continuity of order $\mathrm{n}$ of $\Theta$ defined by

$$
w_{n}(\lambda)=\sup _{x \in R^{m},|\sigma|<\lambda}\left(\int_{S^{m-1}}\left|\Theta\left(x, \sigma t^{\prime}\right)-\Theta\left(x, t^{\prime}\right)\right|^{n} d v\left(t^{\prime}\right)\right)^{\frac{1}{n}},
$$

where $\sigma$ is a rotation in $R^{m}$,

$$
|\sigma|=\sup _{t^{\prime} \in S^{m-1}}\left|\sigma t^{\prime}-t^{\prime}\right| .
$$

The fractional maximal operator with variable kernel is defined by

$$
M_{\Theta, \rho} f(x)=\sup _{n>0} \frac{1}{n^{m-\rho}} \int_{|x-z|<n} f(z)|\Theta(x, x-z)| d z .
$$

The space $L_{l o c}^{p(\cdot)}(E)$ is defined by

$$
L_{l o c}^{p(\cdot)}(E)=\left\{f \text { is measurable }: f \in L^{p(\cdot)}(N) \text { for all compact } N \subset E\right\} .
$$

We denote

$$
p_{-}=e s s \inf \{p(x): x \in E\}, \quad p_{+}=e s s \sup \{p(x): x \in E\} .
$$

Denote $\Gamma(E)$ set of allmeasurable functions $p(\cdot)$ satisfying $p_{-}>1$ and $p_{+}<\infty$. Also denote $\Pi(E)$ the set of all function $p(\cdot) \in \Gamma(E)$ satisfying the $\mathrm{M}$ is bounded on $L^{p(\cdot)}(E)$. Let $B_{i}=\left\{x \in R^{m}:|x| \leq 2^{i}\right\}, C_{i}=B_{i} \backslash B_{i-1}, \chi_{i}=\chi_{C_{i}}, i \in Z$. 


\section{Auxiliary statements and assertions}

Definition 2.1. Let $\beta \in R, 0<q<\infty, p(\cdot) \in \Gamma\left(R^{m}\right)$ and $0 \leq \alpha<\infty$. The Herz-Morrey space with variable exponent $M H_{q, p(\cdot)}^{\beta, \alpha}\left(R^{m}\right)$ is defined by

$$
\begin{aligned}
& M H_{q, p(\cdot)}^{\beta, \alpha}\left(R^{m}\right)=\left\{f \in L_{L o c}^{p(\cdot)}\left(R^{m} \backslash(0)\right):\|f\|_{M H_{q, p(\cdot)}^{\beta, \alpha}\left(R^{m}\right)}<\infty\right\}, \\
& \|f\|_{M H_{q, p(\cdot)}^{\beta, \alpha}\left(R^{m}\right)}=\sup _{L \in Z} 2^{-L \alpha}\left\{\sum_{i=-\infty}^{L} 2^{i \beta p}\left\|f \chi_{i}\right\|_{L^{p(\cdot)}}^{q}\right\}^{\frac{1}{q}} .
\end{aligned}
$$

We set $H_{q(\cdot)}^{\beta, p}\left(R^{m}\right)=M H_{p, q(\cdot)}^{\beta, 0}\left(R^{m}\right)$ (see [9]).

Similarly in [4], let $p(\cdot) \in \Gamma\left(R^{m}\right)$ be such that

$$
\begin{array}{ll}
|p(x)-p(z)| \leq \frac{-C}{\log (|x-z|)}, & |x-z| \leq \frac{1}{2}, \\
|p(x)-p(z)| \leq \frac{C}{\log (e+|x|)}, & |z| \geq|x| .
\end{array}
$$

Proposition $2.1([14])$. Let $p_{1}(\cdot) \in \Pi\left(R^{m}\right), \Theta \in L^{\infty}\left(R^{m}\right) \times L^{n}\left(S^{m-1}\right)$. Let $0<\rho \leq \frac{m}{\left(p_{1}\right)}$, and define the variable exponent $p_{2}(\cdot)$ as

$$
\frac{1}{p_{1}(x)}-\frac{1}{p_{2}(x)}=\frac{\rho}{m}
$$

Then for all $f \in L^{p_{1}(\cdot)}\left(R^{m}\right)$, we have that

$$
\left\|M_{\Theta, \rho} f\right\|_{L^{p_{2}(\cdot)\left(R^{m}\right)}}=\|f\|_{L^{p_{1}(\cdot)}\left(R^{m}\right)} \cdot
$$

Lemma 2.1 ([5]). Let $0<\rho<m, n>1$, satisfies the $L^{n}$-Dini condition. Let there exists an $0<\beta<\frac{1}{2}$ such that for $|z|<\beta_{0} R$ it holds

$$
\left(\int_{R<|x|<2 R}\left|\frac{\Theta(x, x-z)}{|x-z|^{m-\rho}}-\frac{\Theta(x, x)}{|x|^{m-\rho}}\right|^{n} d x\right)^{\frac{1}{n}} \leq C R^{\left(\frac{m}{n}-m+\rho\right)}\left(\frac{|z|}{R}+\int_{\frac{|z|}{2 R}}^{\frac{|z|}{R}} \frac{w_{n}(\lambda)}{\lambda} d \lambda\right) .
$$

Lemma 2.2 ([6]). Let $p(\cdot) \in \Pi\left(R^{m}\right)$ and $0<p^{-} \leq p^{+}<\infty$.

a) For a cube with $|\Delta| \leq 2^{m}$, and all $\chi \in \Delta$, it holds $\left\|\chi_{\Delta}\right\|_{L^{p(\cdot)}}=|\Delta|^{\frac{1}{p(x)}}$;

b) For a cube $|\Delta| \geq 1$, the $\left\|\chi_{\Delta}\right\|_{L^{p(\cdot)}}=|\Delta|^{\frac{1}{p_{\infty}}}$ and where $p_{\infty}=\lim _{x \longrightarrow \infty} p(x)$.

Lemma 2.3 ([15]). Let $p(\cdot) \in \Pi\left(R^{m}\right)$, and there exist a constant $C>0$ such that for all balls $D$ in $R^{m}$, we get

$$
\frac{1}{|D|}\left\|\chi_{D}\right\|_{L^{p(\cdot)\left(R^{m}\right)}} \leq C
$$




\section{Main result}

Theorem 3.1. Let $0<\rho<m, 0<\mu \leq 1, \alpha<\beta<m \lambda_{1}+\mu, 0<q_{1} \leq q_{2}<\infty$. Suppose that $t \in R, \Theta(\cdot, t) \in L^{\infty}\left(R^{m}\right)$ and the integral modulus of continuity $w_{n}(\lambda)$ satisfies

$$
\int_{0}^{1} \frac{w_{n}(\lambda)}{\lambda^{1+\mu}} d \lambda<\infty
$$

And let $p_{1}(\cdot) \in \Pi\left(R^{m}\right)$ satisfy $0<\rho \leq \frac{m}{\left(p_{1}\right)_{+}}$and define the variable exponent $p_{2}(x)$ as $\frac{1}{p_{1}(x)}-\frac{1}{p_{2}(x)}=\frac{\rho}{m}$, then we have for all $f$

$$
\left\|M_{\Theta, \rho} f\right\|_{M H_{q_{2}, p_{2}(\cdot)}^{\beta, \alpha}\left(R^{m}\right)} \leq C\|f\|_{M H_{q_{1}, p_{1}(\cdot)}^{\beta, \alpha}\left(R^{m}\right)} .
$$

Proof. For $\|f\|_{M H_{q_{1}, p_{1}(\cdot)}^{\beta, \alpha}\left(R^{m}\right)}$, we apply inequality

$$
\begin{aligned}
& {\left[\sum_{k=1}^{\infty} \beta_{k}\right]^{\frac{q_{1}}{q_{2}}} \leq \sum_{k=1}^{\infty} \beta_{k}^{\frac{q_{1}}{q_{2}}}, \quad q_{1}, q_{2}>0} \\
& \left\|M_{\Theta, \rho} f\right\|_{M H_{q_{2}, p_{2}(\cdot)}^{\beta_{1}}\left(R^{m}\right)}^{q_{1}}=\sup _{L \in Z} 2^{-L \alpha q_{1}}\left\{\sum_{i=-\infty}^{L} 2^{i \beta q_{2}}\left\|M_{\Theta, \rho}(f) \chi_{i}\right\|_{L^{p_{2}(\cdot)}\left(R^{m}\right)}^{q_{2}}\right\}^{\frac{q_{1}}{q_{2}}} \\
& \leq \sup _{L \in Z} 2^{-L \alpha q_{1}}\left\{\sum_{i=-\infty}^{L} 2^{i \beta q_{1}}\left\|M_{\Theta, \rho}(f) \chi_{i}\right\|_{L^{p_{2}(\cdot)}\left(R^{m}\right)}^{q_{1}}\right\}
\end{aligned}
$$

If we denote

$$
f(x)=\sum_{i=-\infty}^{\infty} f(x) \chi_{i}=\sum_{i=-\infty}^{\infty} f_{i}(x)
$$

then we have

$$
\begin{aligned}
\left\|M_{\Theta, \rho} f\right\|_{M H_{q_{2}, p_{2}(\cdot)}^{q_{1}\left(R^{m}\right)}} \leq \sup _{L \in Z} 2^{-L \alpha q_{1}} \sum_{i=-\infty}^{L} 2^{i \beta q_{1}}\left(\sum_{k=-\infty}^{\infty}\left\|M_{\Theta, \rho}\left(f_{k}\right) \chi_{i}\right\|_{L^{p_{2}(\cdot)}\left(R^{m}\right)}\right)^{q_{1}} \\
\leq \sup _{L \in Z} 2^{-L \alpha q_{1}} \sum_{i=-\infty}^{L} 2^{i \beta q_{1}}\left(\sum_{k=-\infty}^{i-2}\left\|M_{\Theta, \rho}\left(f_{k}\right) \chi_{i}\right\|_{L^{p_{2}(\cdot)}\left(R^{m}\right)}\right)^{q_{1}} \\
\quad+\sup _{L \in Z} 2^{-L \alpha q_{1}} \sum_{i=-\infty}^{L} 2^{i \beta q_{1}}\left(\sum_{k=i-1}^{\infty}\left\|M_{\Theta, \rho}\left(f_{k}\right) \chi_{i}\right\|_{L^{p_{2}(\cdot)}\left(R^{m}\right)}\right)^{q_{1}} \\
=: J_{1}+J_{2} .
\end{aligned}
$$

Let as derive an $J_{1}$ using condition on $f_{k}$. Using Minkowski's inequality for $k \leq i-1$, we 
get

$$
\begin{aligned}
& \left\|M_{\Theta, \rho}\left(f_{k}\right) \chi_{i}\right\|_{L^{p_{2}(\cdot)}\left(R^{m}\right)}=\left(\int_{C_{i}}\left|M_{\Theta, \rho}\left(f_{k}\right)\right|^{p_{2}(\cdot)} d x\right)^{\frac{1}{p_{2}(\cdot)}}, \\
& \left|M_{\Theta, \rho}\left(f_{k}\right)\right|=\left|\int_{R^{m}} \frac{\Theta(x, x-z)}{|x-z|^{m-\rho}} f_{k}(z) d z\right| .
\end{aligned}
$$

Then we have

$$
\begin{aligned}
& \left\|M_{\Theta, \rho}\left(f_{k}\right) \chi_{i}\right\|_{L^{p_{2}(\cdot)}\left(R^{m}\right)} \\
= & \int_{D_{k}} f_{k}(y)\left\|\left|\frac{\Theta(x, x-z)}{|x-z|^{m-\rho}}-\frac{\Theta(x, x)}{|x|^{m-\rho}}\right| \chi_{i}\right\|_{L^{p_{2}(\cdot)}\left(R^{m}\right)} d z .
\end{aligned}
$$

Since $n>p_{2}^{+}, \frac{1}{p_{2}(x)}=\frac{1}{n}+\frac{1}{p_{2}^{\prime}(x)}$ by see [1, Lemma 3.3] and we get

$$
\begin{aligned}
& \left\|\left|\frac{\Theta(x, x-z)}{|x-z|^{m-\rho}}-\frac{\Theta(x, x)}{|x|^{m-\rho}}\right| \chi_{i}\right\|_{L^{p_{2}(\cdot)}\left(R^{m}\right)} \\
\leq & \left\|\frac{\Theta(x, x-z)}{|x-z|^{m-\rho}}-\frac{\Theta(x, x)}{|x|^{m-\rho}}\right\|_{L^{n}\left(R^{m}\right)}\left\|\chi_{i}\right\|_{L^{p_{2}^{\prime}(\cdot)}\left(R^{m}\right)} \\
\leq & \left\|\frac{\Theta(x, x-z)}{|x-z|^{m-\rho}}-\frac{\Theta(x, x)}{|x|^{m-\rho}}\right\|_{L^{n}\left(R^{m}\right)}\left\|\chi_{D_{i}}\right\|_{L^{p_{2}^{\prime}(\cdot)}\left(R^{m}\right)} .
\end{aligned}
$$

According to Lemma 2.2 and $\frac{1}{p_{2}(x)}-\frac{1}{n}=\frac{1}{p_{2}^{\prime}(x)}$, then we have

$$
\left\|\chi_{D_{i}}\right\|_{L^{p_{2}^{\prime}(\cdot)\left(R^{m}\right)}} \approx\left\|\chi_{D_{i}}\right\|_{L^{p_{1}(\cdot)\left(R^{m}\right)}}|D|^{\frac{-1}{n} \frac{\rho}{m}} .
$$

Combining Lemma 2.1 and $2^{k-i} \leq 2^{(k-i) \mu}$, we get

$$
\begin{aligned}
& \left\|\frac{\Theta(x, x-z)}{|x-z|^{m-\rho}}-\frac{\Theta(x, x)}{|x|^{m-\rho}}\right\|_{L^{n}\left(R^{m}\right)} \\
\leq & C R^{\left(\frac{m}{n}-m+\rho\right)}\left(\frac{|z|}{2^{i-1}}+\int_{2^{i}}^{2^{i-1}} \frac{w_{n}(\lambda)}{\lambda} d \lambda\right) \\
\leq & C R^{\left(\frac{m}{n}-m+\rho\right)}\left(2^{k-i}+2^{(k-i) \mu} \int_{0}^{1} \frac{w_{n}(\lambda)}{\lambda^{1+\mu}} d \lambda\right) \\
\leq & C R^{\left(\frac{m}{n}-m+\rho\right)} 2^{(k-i) \mu}\left(1+\int_{0}^{1} \frac{w_{n}(\lambda)}{\lambda^{1+\mu}} d \lambda\right) \\
\leq & C 2^{(i-1)\left(\frac{m}{n}-m+\rho\right)} 2^{(k-i) \mu} .
\end{aligned}
$$

It follows that

$$
\left\|M_{\Theta, \rho}\left(f_{k}\right) \chi_{i}\right\|_{L^{p_{2}(\cdot)\left(R^{m}\right)}} \leq C 2^{-i m+(k-i) \mu} \int_{D_{k}} f_{k}(z) d z\left\|\chi_{D_{i}}\right\|_{L^{p_{1}(x)}\left(R^{m}\right)} .
$$


Using (see [1, Lemma 3.1 and Lemma 3.5]) and Lemma 2.3, it follows that

$$
\begin{aligned}
& \left\|M_{\Theta, \rho}\left(f_{k}\right) \chi_{i}\right\|_{L^{p_{2}(\cdot)}\left(R^{m}\right)} \\
\leq & C 2^{-i m+(k-i) \mu}\left\|f_{k}\right\|_{L^{p_{1}(x)}\left(R^{m}\right)}\left\|\chi_{D_{k}}\right\|_{L^{p_{1}^{\prime}(\cdot)}\left(R^{m}\right)}\left\|\chi_{D_{i}}\right\|_{L^{p_{1}(x)}\left(R^{m}\right)} \\
\leq & C 2^{(k-i) \mu}\left\|f_{k}\right\|_{L^{p_{1}(x)}\left(R^{m}\right)}\left\|\chi_{D_{k}}\right\|_{L^{p_{1}^{\prime}(\cdot)}\left(R^{m}\right)} 2^{-i m}\left\|\chi_{D_{i}}\right\|_{L^{p_{1}(x)}\left(R^{m}\right)} \\
\leq & C 2^{(k-i) \mu}\left\|f_{k}\right\|_{L^{p_{1}(x)}\left(R^{m}\right)} \frac{\left\|\chi_{D_{k}}\right\|_{L^{p_{1}^{\prime}(\cdot)}\left(R^{m}\right)}}{\left\|\chi_{D_{i}}\right\|_{L^{p_{1}(x)}\left(R^{m}\right)}} \\
\leq & C 2^{(k-i) \mu} 2^{(k-i) m \lambda_{1}}\left\|f_{k}\right\|_{L^{p_{1}(x)}\left(R^{m}\right)} \\
\leq & 2^{(k-i)\left(\mu+m \lambda_{1}\right)}\left\|f_{k}\right\|_{L^{p_{1}(x)}\left(R^{m}\right)} .
\end{aligned}
$$

Hence we have

$$
\begin{aligned}
J_{1} & \leq C \sup _{L \in Z} 2^{-L \alpha q_{1}} \sum_{i=-\infty}^{L}\left(\sum_{k=-\infty}^{i-2} 2^{(k-i)\left(\mu+m \lambda_{1}\right)}\left\|f_{k}\right\|_{L^{p_{1}(x)}\left(R^{m}\right)}\right)^{q_{1}} \\
& \leq C \sup _{L \in Z} 2^{-L \alpha q_{1}} \sum_{i=-\infty}^{L}\left(\sum_{k=-\infty}^{i-2} 2^{\beta k} 2^{(k-i)\left(\mu+m \lambda_{1}-\beta\right)}\left\|f_{k}\right\|_{L^{p_{1}(x)}\left(R^{m}\right)}\right)^{q_{1}} .
\end{aligned}
$$

We consider the cases $1<q_{1}<\infty$ and $0<q_{1} \leq 1$.

If $0<q_{1} \leq 1$, using the Holder's inequality, we have

$$
\begin{aligned}
& J_{1} \leq C \sup _{L \in Z} 2^{-L \alpha q_{1}} \sum_{i=-\infty}^{L}\left(\sum_{k=-\infty}^{i-2} 2^{\beta k q_{1}} 2^{(k-i)\left(\mu+m \lambda_{1}-\beta\right)^{\frac{q_{1}}{2}}}\left\|f_{k}\right\|_{L^{p_{1}(x)}\left(R^{m}\right)}^{q_{1}}\right) \\
& \times\left(\sum_{k=-\infty}^{i-2} 2^{\frac{(k-i)\left(\mu+m \lambda_{1}-\beta\right)^{\prime}}{2}}\right)^{\frac{q_{1}}{q_{1}^{\prime}}} \\
& \leq C \sup _{L \in Z} 2^{-L \alpha q_{1}} \sum_{i=-\infty}^{L} \sum_{k=-\infty}^{i-2} 2^{\beta k q_{1}} 2^{(k-i)\left(\mu+m \lambda_{1}-\beta\right)^{\frac{q_{1}}{2}}}\left\|f_{k}\right\|_{L^{p_{1}(x)}\left(R^{m}\right)}^{q_{1}} \\
& \leq C \sup _{L \in Z} 2^{-L \alpha q_{1}} \sum_{k=-\infty}^{i-2} 2^{\beta k q_{1}}\left\|f_{k}\right\|_{L^{p_{1}(x)}\left(R^{m}\right)}^{q_{1}} \sum_{i=k+2}^{L} 2^{(k-i)\left(\mu+m \lambda_{1}-\beta\right)^{\frac{q_{1}}{2}}} \\
& \leq C \sup _{L \in Z} 2^{-L \alpha q_{1}} \sum_{k=-\infty}^{i-2} 2^{\beta k q_{1}}\left\|f_{k}\right\|_{L^{p_{1}(x)}\left(R^{m}\right)}^{q_{1}} \\
& \leq C\|f\|_{M H_{q_{1}, p_{1}(\cdot)}^{\beta_{1}\left(R^{m}\right)}}^{q_{1}}
\end{aligned}
$$


If $0<q_{1} \leq 1, \beta<m \lambda_{1}+\mu$, we get

$$
\begin{aligned}
J_{1} & \leq C \sup _{L \in Z} 2^{-L \alpha q_{1}} \sum_{i=-\infty}^{L} \sum_{k=-\infty}^{i-2} 2^{\beta k q_{1}} 2^{(k-i)\left(\mu+m \lambda_{1}-\beta\right)^{q_{1}}}\left\|f_{k}\right\|_{L^{p_{1}(x)}\left(R^{m}\right)}^{q_{1}} \\
& \leq C \sup _{L \in Z} 2^{-L \alpha q_{1}} \sum_{k=-\infty}^{i-2} 2^{\beta k q_{1}}\left\|f_{k}\right\|_{L^{p_{1}(x)}\left(R^{m}\right)}^{q_{1}} \sum_{i=k+2}^{L} 2^{(k-i)\left(\mu+m \lambda_{1}-\beta\right)^{q_{1}}} \\
& \leq C \sup _{L \in Z} 2^{-L \alpha q_{1}} \sum_{k=-\infty}^{i-2} 2^{\beta k q_{1}}\left\|f_{k}\right\|_{L^{p_{1}(x)}\left(R^{m}\right)}^{q_{1}} \\
& \leq C\|f\|_{M H_{q_{1}, p_{1}(\cdot)}^{\beta, \alpha}\left(R^{m}\right)}^{q_{1}}
\end{aligned}
$$

Now, we estimate $J_{2}$, by using Proposition 2.1, we have

$$
\begin{aligned}
J_{2} \leq C \sup _{L \in Z} 2^{-L \alpha q_{1}} \sum_{i=-\infty}^{L} 2^{\beta i q_{1}}\left(\sum_{k=i-1}^{\infty}\left\|M_{\Theta, \rho}\left(f_{k}\right) \chi_{i}\right\|_{L^{p_{2}(\cdot)}\left(R^{m}\right)}\right)^{q_{1}} \\
\leq C \sup _{L \in Z} 2^{-L \alpha q_{1}} \sum_{i=-\infty}^{L} 2^{\beta i q_{1}}\left(\sum_{k=i-1}^{\infty}\left(\left\|f_{k}\right\|_{L^{p_{1}(\cdot)}\left(R^{m}\right)}\right)^{q_{1}}\right. \\
\leq C \sup _{L \in Z} 2^{-L \alpha q_{1}} \sum_{i=-\infty}^{L}\left(\sum_{k=i-1}^{\infty} 2^{\beta k} 2^{(i-k) \beta}\left\|f_{k}\right\|_{L^{p_{1}(\cdot)}\left(R^{m}\right)}\right)^{q_{1}} \\
\leq C \sup _{L \in Z} 2^{-L \alpha q_{1}} \sum_{i=-\infty}^{L}\left(\sum_{k=i-1}^{i+1} 2^{\beta k} 2^{(i-k) \beta}\left\|f_{k}\right\|_{L^{p_{1}(\cdot)}\left(R^{m}\right)}\right)^{q_{1}} \\
\quad+C \sup _{L \in Z} 2^{-L \alpha q_{1}} \sum_{i=-\infty}^{L}\left(\sum_{k=i+2}^{\infty} 2^{\beta k} 2^{(i-k) \beta}\left\|f_{k}\right\|_{L^{p_{1}(\cdot)}\left(R^{m}\right)}\right)^{q_{1}} \\
=: J_{1}+J_{2} .
\end{aligned}
$$

Then we have,

$$
\begin{aligned}
& \leq C \sup _{L \in Z} 2^{-L \alpha q_{1}} \sum_{i=-\infty}^{L}\left(\sum_{k=i-1}^{i+1} 2^{\beta k} 2^{(i-k) \beta}\left\|f_{k}\right\|_{L^{p_{1}(\cdot)}\left(R^{m}\right)}\right)^{q_{1}} \\
& \leq C \sup _{L \in Z} 2^{-L \alpha q_{1}} \sum_{i=-\infty}^{L} 2^{\beta i q_{1}}\left\|f_{k}\right\|_{L^{p_{1}(\cdot)}\left(R^{m}\right)}^{q_{1}} \\
& \leq C\|f\|_{M H_{q_{1}, p_{1}(\cdot)}^{\left.q_{1}, R^{m}\right)}} \cdot
\end{aligned}
$$

Estimate $J_{2}$, for $\alpha<\beta$,

$$
J_{2} \leq C \sup _{L \in Z} 2^{-L \alpha q_{1}} \sum_{i=-\infty}^{L}\left(\sum_{k=i+2}^{\infty} 2^{\beta k} 2^{(i-k) \beta}\left\|f_{k}\right\|_{L^{p_{1}(\cdot)}\left(R^{m}\right)}\right)^{q_{1}}
$$




$$
\begin{aligned}
& \leq C \sup _{L \in Z} \sum_{i=-\infty}^{L} 2^{(i-L) \alpha q_{1}}\left(\sum_{k=i+2}^{\infty} 2^{\beta k^{(i-k)(\beta-\alpha)}} 2^{-k \alpha}\left\|f_{k}\right\|_{L^{p_{1}(\cdot)}\left(R^{m}\right)}\right)^{q_{1}} \\
& \leq C \sup _{L \in Z} \sum_{i=-\infty}^{L} 2^{(i-L) \alpha q_{1}}\left(\sum_{k=i+2}^{\infty} 2^{(i-k)(\beta-\alpha)} 2^{-k \alpha}\left\{\sum_{m=-\infty}^{i} 2^{m \beta}\left\|f_{k}\right\|_{L^{p_{1}(\cdot)}\left(R^{m}\right)}\right\}^{\frac{1}{q_{1}}}\right)^{q_{1}} \\
& \leq C \sup _{L \in Z} \sum_{i=-\infty}^{L} 2^{(i-L) \alpha q_{1}}\left(\sum_{k=i+2}^{\infty} 2^{(i-k)(\beta-\alpha)}\right)^{q_{1}}\|f\|_{M H_{q_{1}, p_{1}(\cdot)}^{q_{1}}\left(R^{m}\right)}^{q_{1}} \\
& \leq C\|f\|_{M H_{q_{1}, p_{1}(\cdot)}^{q_{1}}\left(R^{m}\right)}^{q^{\beta}} .
\end{aligned}
$$

Proof of Theorem 3.1 was completed.

\section{Conclusions}

We obtained the boundedness of fractional maximal operator with variable kernel on the Herz-Morrey spaces by a new method.

\section{Acknowledgements}

Author thanks referee for his/her valuable suggestion remarks regarding the manuscript.

\section{References}

[1] A. Abdalmonem, O. Abdalrhman and S. Tao, The Boundedness of fractional integral with variable kernel on variable exponent Herz-Morrey spaces, J. Appl. Math. Phys., 4 (2016), 787-795.

[2] A. Abdalmonem, O. Abdalrhman and S. Tao, Boundedness of fractional integral with variable kernel and Their commutators on variable exponent Herz spaces, Appl. Math., 7 (2016), $1165-1182$.

[3] A. Abdalmonem, O. Abdalrhman and S. Tao, Boundedness of fractional Marcinkiewicz integral with variable kernel on variable exponent Morrey-Herz spaces, Open J. Math. Sci., 2(1) (2018), 93-114

[4] D. Cruz-Uribe and A. Fiorenza, Variable Lebesgue Spaces, Foundations and Harmonic Analysis, Springer, (2013), New York.

[5] D. Cruz-Uribe, A. Fiorenza, J. Martell and C. Pe'rez, The boundedness of classical operators on variable $L_{p}$ spaces, Annales Academiae Scientiarum Fennicae Mathematica, 31 (2006), 239-264.

[6] Y. Ding and S. Lu, Homogeneous Fractional Integrals on Hardy spaces, Tohoku Math. J., 52 (2000), 153-162.

[7] M. Izuki, Boundedness of vector-valued sublinear operators on Herz-Morrey spaces with variable exponent, Mathematical Sciences Research J., 13 (2009), 243-253.

[8] M. Izuki, Herz and amalgam spaces with variable exponent, the haar wavelets and greediness of the wavelet system, East J. Approx., 15 (2009), 87-109. 
[9] M. Izuki, Boundedness of commutators on herz spaces with variable exponent, Rendiconti del Circolo Matematico di Palermo, 59 (2010), 199-213.

[10] M. Izuki, Fractional integrals on Herz-Morrey spaces with variable exponent, Hiroshima Math. J., 40 (2010), 343-355.

[11] I. F. Mamedov, Y. Zeren and L. Akin, Compactification of weighted Hardy operator in variable exponent Lebesgue spaces, Asian J. Math. Computer Res., 17(1) (2017), 38-47.

[12] S. Lu and L. Xu, Boundedness of rough singular integral operators on the homogeneous Morrey-Herz spaces, Hokkaido Math. J., 34 (2005), 299-314.

[13] Z. Wang and Y. Zhu, Boundedness of multilinear fractional integral operators on HerzMorrey spaces with variable exponent, Journal of Lantong University (Natural Science Edition), 13 (2014), 60-68.

[14] $\mathrm{H}$. Wu and J. Lan, The boundedness for a class of rough fractional integral operators on variable exponent Lebesgue spaces, Analysis in Theory and Applications, 28 (2012), 286293.

[15] J. Tan and Z. Liu, Some boundedness of homogeneous fractional integrals on variable exponent function spaces, ACTA Mathematics Science (Chinese Series), 58 (2015), 310-320.

[16] L. Diening, P. Hasto, P. Harjulehto and M. M. Ruzicka, Lebesgue and Sobolev Spaces with Variable Exponents, Springer-Verlag, (2011).

[17] E. Piskin, Finite time blow up of solutions for a strongly damped nonlinear Klein-Gordon equation with variable exponents, Honam Math. J., 40(4) (2018), 771-783. 